\title{
Correspondence- Technical Note: Video laryngoscope-guided (GlideScope (B) injection of botulinum toxin in laryngectomy patients with limited neck extension
}

\author{
Yadsan Devabalan ${ }^{1}$, Akshat Malik ${ }^{1}$, and Peter Clarke ${ }^{2}$ \\ ${ }^{1}$ Charing Cross Hospital \\ ${ }^{2}$ Imperial College Healthcare NHS Trust
}

June 8, 2020

\begin{abstract}
Functional rehabilitation of post-laryngectomy patients is a challenging task; this includes achieving optimal speech and swallowing outcomes. Botox injections are utilised to improve pharyngoesophageal segment tonicity to improve speech and swallowing outcomes. We propose a novel and efficacious technique using a portable video-laryngoscope, GlideScopeß, to directly visualise the injection of botulinum toxin in total laryngectomy patients with severe radiation-induced changes.
\end{abstract}

Key points:

Botox injections are utilised to improve pharyngoesophageal segment tonicity to improve speech and swallowing outcomes

Video-laryngoscope guided injection of Botox into the neopharynx is a easy and safe technique

It is particularly useful for post-radiotherapy patients with limited neck extension where rigid direct pharyngoscopy is impossible

Introduction:

Functional rehabilitation of post-laryngectomy patients is a challenging task; this includes achieving optimal speech and swallowing outcomes. Common problems in these patients relate to either the hypertonicity of the pharyngoesophageal (PE) segment, PE segment spasm or stricture formation ${ }^{(1)}$. Many of these patients would have received radiotherapy/chemo-radiotherapy as a definitive therapy (before surgery) or as adjuvant therapy, and radiation induced fibrosis further worsens the functional outcomes. Inadequate myotomy probably increases the risk of hypertonicity and spasm.

Pharyngeal constrictor myotomy ${ }^{(2)}$, pharyngeal plexus neurectomy and various methods of surgical closure at the time of the operation ${ }^{(1)}$ can all mitigate the risk of these complications. Once established, stenosis can be treated by dilatation with balloon or bougie, or augmenting the neopharynx surgically. Hypertonicity and spasm, however, can be treated with the use of Botulinum toxin (Botox) injections, which has been shown to be efficacious ${ }^{(3)}$. This chemically denervates the hypertonic pharyngoesophageal segment by blocking the release of acetylcholine at the neuromuscular junction. This reduced tone can lead to permanent improvements in speech in the majority of patients. These are usually injected under videofluoroscopic control but in patients with significant fibrosis it can be difficult to get sufficient injection directly into the muscle fibres and so is undertaken under direct vision under general anaesthetic, including if an anaesthetic is required for another procedure such as dilatation or secondary puncture. ${ }^{(4)}$ Other methods of injecting botulinum toxin include electromyographic (EMG) guided $^{(2)}$ and ultrasound-guided. However, rigid endoscopy guided 
injection of the Botox remains a helpful approach in some patients with failed prior transcutaneous injections. This can however prove difficult in post-radiotherapy patients, particularly those with limited neck extension.

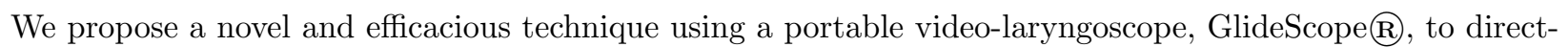
ly visualise the injection of botulinum toxin in total laryngectomy patients with severe radiation-induced changes. This gives a more stable view than that from a flexible laryngoscope.

Technical Description:

6 patients were treated over the last year using this method. General anaesthesia was given to these patients and they were ventilated through an armoured tube inserted through the laryngeal stoma. Patients were positioned with their neck flexed and head extended, though the extension was limited in these patients due to post-radiotherapy fibrosis. A GlideScope was inserted through the oral cavity along the tongue and the top of the scope was used to lift the base of the tongue (figure 1). This provided a good view of the neopharynx in all the cases. Botox was kept ready pre-loaded on a syringe with a 22 -gauge needle. The needle was inserted through the neck and under the direct vision of the GlideScope, Botox was injected into the $\mathrm{PE}$ segment/posterior pharyngeal wall at the level of the neopharynx down to the level of the valve (figure 2). It was feasible to inject Botox in all the patients using this technique and in none of them did we have to resort to any other technique. All of them had improvements in their voice outcomes.

Discussion:

GlideScope $\left(\mathrm{B}\right.$ is a video laryngoscope instrument that is primarily used for oral endotracheal intubation ${ }^{(5)}$. It provides optimal glottic views and it is this benefit that can be directly translated into our patients to provide a good view of the neopharynx. The main benefit with this is that it allows for an optimal view without the need for substantial neck extension (figure 2), which is very difficult to achieve in patients with extensive post-radiotherapy fibrosis.

Usually, video fluoroscopy-guided Botox injections are performed. However, in some patients injections are required under general anaesthetic, which has the advantage of excellent visual control and minimal risk to blood vessels or surrounding muscles. ${ }^{(4)}$ Usually a view of the neopharynx is then achieved with a rigid pharyngoscope, but in those with short necks or cervical spine pathologies, it is difficult to extend the neck to allow a good view and provide access. Video fluoroscopy may not be available in a resource-limited setting and requires a radiographer and the Speech and Language therapist to be present. There is the added risk of radiation exposure, particularly if these patients require repeat procedures.

Rigid instruments such as pharyngoscopes and oesophagoscopes ${ }^{(4)}$ are used to directly visualise and inject the Botox. However, it is challenging to use rigid instruments in patients with limited neck extension, these further carry a risk of perforation to the surrounding tissue, something that does not exist with the GlideScope. Furthermore, video-laryngoscopy carries a significantly lower risk of aerosol-generation and transmission compared to direct laryngoscopies ${ }^{(6)}$, an issue that is topical in the COVID-19 pandemic.

The GlideScope (B) can also provide direct vision of the neopharynx where this is impossible with a rigid instrument to enable accurate placement of dilation balloons or to ensure the safe passing of bougies and has been used by the authors for this purpose.

\section{Conclusion:}

We present a novel use for video-laryngoscope to enable ENT surgeons to safely, easily and efficaciously administer Botulinum toxin injections in post-laryngectomy patients having a hypertonic/spasmodic PE segment.

1. Bayles SW, Deschler DG. Operative prevention and management of voice-limiting pharyngoesophageal spasm. Otolaryngol Clin North Am. 2004;37(3):547-58. 
2. Chao S-S, Graham SM, Hoffman HT. Management of pharyngoesophageal spasm with Botox. Otolaryngologic Clinics of North America. 2004;37(3):559-66.

3. Lightbody KA, Wilkie MD, Kinshuck AJ, Gilmartin E, Lewis-Jones H, Jones TM, et al. Injection of botulinum toxin for the treatment of post-laryngectomy pharyngoesophageal spasm-related disorders. Ann R Coll Surg Engl. 2015;97(7):508-12.

4. Krause E, Hempel JM, Gürkov R. Botulinum toxin A prolongs functional durability of voice prostheses in laryngectomees with pharyngoesophageal spasm. Am J Otolaryngol. 2009;30(6):371-5.

5. Russell T-M, Hormis A, Trust RNF. Should the Glidescope video laryngoscope be used first line for all oral intubations or only in those with a difficult airway? A review of current literature. Journal of perioperative practice. 2018;28(12):322-33.

6. Zeidan A, Bamadhaj M, Al-Faraidy M, Ali M. Videolaryngoscopy Intubation in Patients with COVID-19: How to Minimize Risk of Aerosolization? Anesthesiology. 2020.

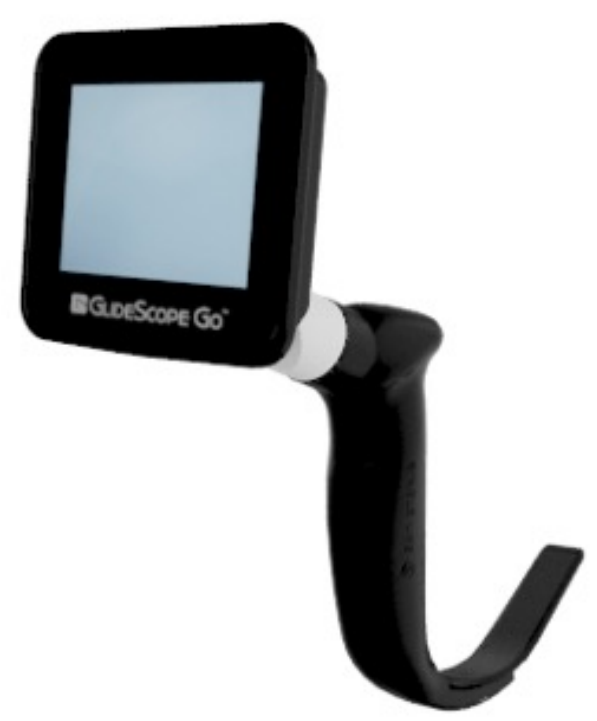

\section{Figure 1: hand-held video laryngoscope, GlideScope ${ }^{\circledR}$}




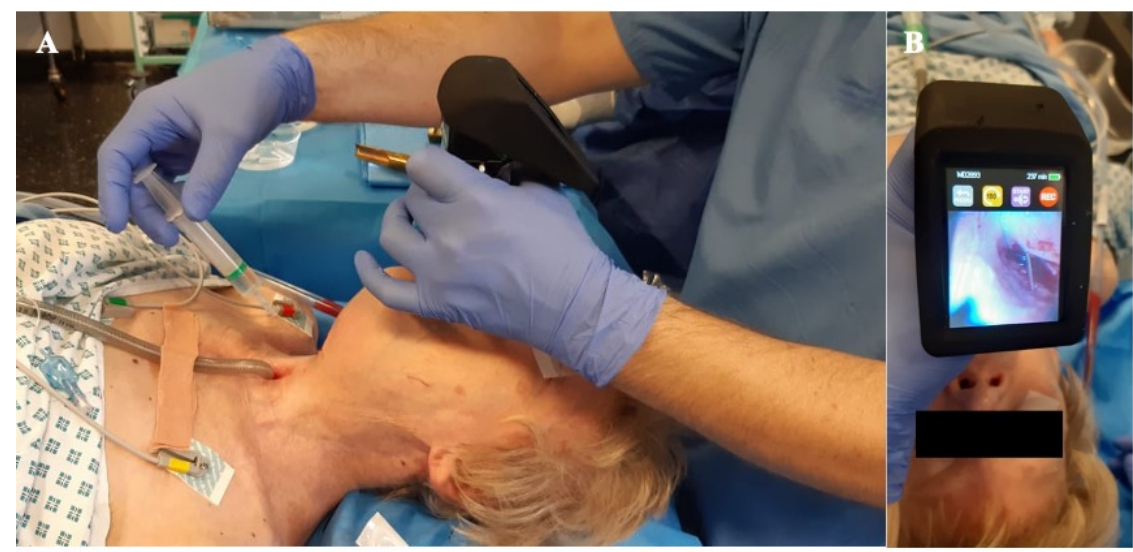

Figure 2: (A) Technique of Botox injection using GlideScope ${ }^{\bullet}$. (B) Direct visualisation of the neopharynx (needle visible in the lumen) using the GlideScope ${ }^{\star}$. 